\title{
Genre et travail indépendant
}

Divisions sexuées et places des femmes dans le non-salariat

\section{Sarah Abdelnour, Sophie Bernard et Julien Gros}

\section{CpenEdition}

\section{Journals}

Édition électronique

URL : http://journals.openedition.org/travailemploi/7459

DOI : 10.4000/travailemploi.7459

ISSN : 1775-416X

Éditeur

DARES - Ministère du Travail

Édition imprimée

Date de publication : 1 avril 2017

Pagination : 5-23

ISSN : 0224-4365

Référence électronique

Sarah Abdelnour, Sophie Bernard et Julien Gros, « Genre et travail indépendant », Travail et Emploi [En ligne], 150 | avril-juin 2017, mis en ligne le 11 juillet 2019, consulté le 23 septembre 2020. URL : http:// journals.openedition.org/travailemploi/7459; DOI : https://doi.org/10.4000/travailemploi.7459 


\title{
Introduction
}

\section{Genre et travail indépendant}

\section{Divisions sexuées et places des femmes dans le non-salariat}

\author{
Sarah Abdelnour ${ }^{*}$, Sophie Bernard ${ }^{* *}$, Julien Gros ${ }^{* * *}$
}

$\mathrm{T}$ out au long du $\mathrm{Xx}^{\mathrm{e}}$ siècle, les femmes n'ont jamais représenté plus de $40 \%$ des non-salarié·e·s, et toujours moins d'un quart des employeur·euse·s. Minoritaires, ces femmes non salariées sont aussi mal connues. La sociologie du genre n'a pourtant pas été aveuglée par le salariat, bien au contraire. Des premières enquêtes ont été menées dans les années 1970 sur les secteurs de l'agriculture et de l'artisanat, mettant en lumière l'imbrication de l'activité économique indépendante et de l'économie domestique. Plus largement, les études de genre ont questionné les frontières de la notion de travail à partir de la mise en évidence du travail domestique et surtout de la division sexuée entre travail productif et reproductif (BERENI et al., 2008). Mais le développement de ces travaux a également été contemporain de la salarisation et de la tertiarisation. Et ainsi, le travail indépendant n'a dès lors plus toujours constitué une zone de recherche privilégiée des études féministes. Au vu des transformations importantes des secteurs artisanal et agricole et de l'essor contemporain de nouvelles formes de travail indépendant, ce dossier entend revisiter les divisions sexuées du non-salariat et les places que les femmes y occupent.

Les transformations récentes de l'indépendance vont de pair avec une redistribution des positions entre femmes et hommes. Si les femmes restent nettement minoritaires dans le groupe composite du non-salariat, des évolutions et des recompositions méritent toutefois que l'on y prête attention. D'une part, dans les secteurs, en déclin, de l'agriculture comme de l'artisanat, les femmes occupent une place croissante, qu'il s'agisse de la formalisation de leur travail au sein d'entreprises familiales ou de création d'activités plus autonomes. D'autre part, les femmes participent également au renouveau récent du travail indépendant, leur présence suivant alors l'hétérogénéité

\footnotetext{
* Université Paris Dauphine, PSL Research University, CNRS, UMR 7170, Institut de recherche interdisciplinaire en sciences sociales (Irisso) ; sarah.abdelnour@ dauphine.fr.

** Université Paris Dauphine, PSL Research University, CNRS, UMR 7170, Irisso ; sophie.bernard@ dauphine.fr.

*** Université Paris Dauphine, PSL Research University, CNRS, UMR 7170, Irisso ; Laboratoire de sociologie quantitative (Crest/Génés) ; juliendgros@gmail.com.
} 
de l'indépendance elle-même, avec l'essor de deux segments distincts : les professions libérales et les auto-entrepreneur-euse-s. Comment dès lors repenser les rapports de genre dans le cadre dynamique des recompositions de l'indépendance ? En quoi les places des femmes s'y trouvent-elles transformées? Entre sociologie de l'emploi et du travail, et dans la lignée des travaux de sociologie du travail et du genre, ce dossier donne à voir l'émergence de nouvelles figures féminines du travail non salarié et permet de documenter les formes d'investissement spécifiques des femmes dans l'indépendance, ainsi que les mutations des rapports de genre qui en découlent.

La percée des femmes dans les bastions masculins de l'indépendance pourrait être envisagée de prime abord comme le signe d'une atténuation des clivages sexués. Les femmes verraient leur place mieux reconnue et encadrée, accéderaient à des positions privilégiées au sein des professions libérales, voire à des positions de cheffes d'entreprise. Mais cette place nouvelle occupée par les femmes dans le monde de l'indépendance correspond-elle toujours à des formes d'égalisation des conditions, voire d'émancipation, ou annonce-t-elle également de nouvelles fragilités et la recomposition d'inégalités genrées ? En effet, il semble que les femmes pourraient particulièrement pâtir de certains aspects de l'indépendance professionnelle. D'abord, ces activités reposent encore souvent sur la mobilisation familiale, rejouant alors les inégalités sexuées du travail domestique et familial, dans les cas où l'entreprise suppose une « cause commune » au niveau de la maisonnée (GolLAC, 2003), y compris quand les femmes sont elles-mêmes indépendantes. Ensuite, et plus largement, le travail indépendant se définit par sa moindre régulation statutaire, ses fortes inégalités internes, des horaires de travail étendus et une grande porosité des espaces et des temps professionnels et domestiques. L'interpénétration du professionnel et du privé peut-elle alors être mise au service d'une « conciliation » plus égalitaire des temps ou participe-t-elle à des formes redoublées d'inégalités dans l'indépendance?

Dans cette introduction, nous reviendrons d'abord sur l'héritage des enquêtes pionnières des années 1970 et 1980, qui ont mis en lumière les fortes inégalités structurant les entreprises familiales, dans lesquelles les hommes étaient les travailleurs indépendants visibles, tandis que les femmes accomplissaient le travail gratuit, domestique comme professionnel. Puis nous décrirons les grandes tendances d'évolution du monde de l'indépendance durant les trente dernières années et plus particulièrement la place qu'y prennent les femmes, entre net recul des aides familiales et essor contrasté de leur place comme indépendantes. Nous formulerons enfin les questions traitées dans le dossier, notamment sur les outils d'analyse des inégalités sexuées dans le non-salariat et présenterons un parcours de lecture des articles qui le composent. 


\section{Indépendance des hommes et travail gratuit des femmes dans les années 1970}

La question des rapports de genre est apparue dès les premières grandes enquêtes de sociologie sur le travail indépendant, au travers de la prise en compte de la dimension conjugale et familiale du travail, productif comme domestique. Les rapports sociaux de sexe observés alors étaient fortement clivés, puisque les hommes étaient systématiquement à la tête de l'entreprise, quand les femmes étaient des aides familiales ${ }^{1}$. Il n'est pas fortuit que ce soit à partir de l'observation des ménages agricoles que Christine DELPHY (1998) ait élaboré une grille d'analyse féministe de la répartition du travail et de la disjonction entre travail productif rémunéré (des hommes) et travail reproductif gratuit (des femmes). Alors qu'être agriculteur est un métier, être agricultrice est appréhendé dans ce cadre comme une simple extension des activités ménagères et n'est pas reconnu comme une activité professionnelle. C'est pourquoi ce secteur constitue pour les théories féministes « une situation d'expérimentation naturelle » (DELPHY, 1983, p. 8). Cette dernière permet, d'une part, de mettre à l'épreuve les théories qui, à partir des écrits de Karl Marx sur la valeur de la force de travail, concluent à l'absence de valeur du travail ménager. D'autre part, elle remet en cause celles qui postulent la moindre participation des femmes à la sphère productive et leur cantonnement à la sphère familiale comme la cause de leur infériorité sociale. L'étude de ce secteur permet ainsi de dévoiler que toutes les femmes mariées sont soumises dans « le mode de production domestique » (ibid., p. 12) à une forme d'exploitation patriarcale visant à leur extorquer du travail gratuit, le lien conjugal étant considéré comme un contrat de servage et la famille comme un «système de classes » (ibid., p. 12). Tandis que l'appropriation du travail des femmes est totale dans le cadre des entreprises familiales, elle est partielle lorsque celles-ci sont salariées et travaillent à l'extérieur, se limitant alors au travail domestique et à « l'élevage » des enfants. L'agriculture n'est ni un rapport familial, ni un rapport de travail, mais « le choc entre les deux » (BARTHEZ, 1982, p. 181). Les femmes agricultrices sont ainsi doublement assujetties : en tant que femmes et en tant que travailleuses, en tant que catégorie sexuelle et en tant que classe sociale (LAGRAVE, 1983). Elles s'inscrivent dans un « métier de couple » qui se caractérise par l'absence de reconnaissance professionnelle des femmes.

Pour reprendre le titre d'un article d'Isabelle BERTAUX-WiAME (2004) à propos d'un modèle qu' elle qualifie de «traditionnel » parmi les artisans et commerçants, devenir indépendant est en effet « une affaire de couple ». La mise à son compte est vécue comme un projet de mobilité sociale qui implique toute la famille. Les travaux sur l'indépendance ont pour point commun de « considérer le couple, et non plus l'individu, comme agent économique » (ZALC, 2005, p. 52). Si les femmes sont minoritaires parmi les travailleur-euse-s indépendant·e-s, elles ont néanmoins toujours travaillé dans les entreprises familiales, mais sans être comptabilisées comme indépendantes

1. Notons en effet que les enquêtes dont nous disposons portent exclusivement sur des couples hétérosexuels. 
(BAtTAgliola, 2000 ; Schweitzer, 2002). Le monde du travail indépendant est en apparence un milieu d'hommes, mais quand on questionne les conditions d'accès aux fonctions de chef.fe d'entreprise et l'organisation quotidienne de l'activité entrepreneuriale, le travail féminin se révèle, ainsi que l'imbrication des rapports familiaux et des activités économiques. Celle-ci a été mise en évidence dans les petites entreprises par des enquêtes sociologiques consacrées à certaines professions indépendantes de l'agriculture, de l'artisanat et du petit commerce (GRESLE, 1981 ; BARTHEZ, 1982 ; ZARCA, 1986 ; MAZAUD, 2013). En mettant au jour cette imbrication, ces travaux ont eu le mérite de permettre un décloisonnement des sociologies du travail et de la famille et de poser les jalons d'un questionnement en termes de genre. Perspective allant presque de soi pour l'étude du travail indépendant, elle ne s'imposait en effet pas spontanément pour l'analyse du salariat, qui s'en distingue par une séparation formelle entre espace-temps productif et espace-temps reproductif ${ }^{2}$. C'est d'ailleurs en questionnant cette frontière par les pratiques de « travail à-côté » d'ouvriers (WEBER, 1989), que Florence WEBER a été amenée à préciser le concept d'économie domestique (TÉNÉDOS, WEBER, 2006).

La participation féminine à l'activité économique exercée par un conjoint indépendant peut ainsi prendre diverses formes, qui incluent la participation explicite à l'activité productive comme la prise en charge des tâches domestiques et familiales. Ces modes de participation ne sont pas figés et peuvent connaître d'autres formes au fil du temps, en fonction des évolutions de l'entreprise et de celles de la famille. I. BERTAUX-Wiame (2004) identifie trois configurations possibles. Dans la première, qualifiée de « traditionnelle », le couple travaille ensemble dans l'entreprise familiale. Ainsi, l'une des conditions d'installation pour un artisan-boulanger est d'avoir une épouse prête à s'investir dans l'affaire et à tenir la boutique pendant qu'il s'active au fournil (BERTAUX-WIAME, 1982). Installation et mariage vont de pair, l'épouse se trouvant alors dans l'obligation de renoncer à ses projets professionnels pour soutenir le projet d'installation de son mari. Dans la deuxième configuration, celle $\mathrm{du}$ « couple conjugal », le mari est indépendant et la femme officiellement inactive. La division sexuée du travail domestique est souvent plus radicale dans le travail indépendant que dans d'autres configurations professionnelles de couple, notamment quand les conjoints sont salariés. L'important investissement au travail du mari justifie alors l'inactivité de l'épouse avec un partage du travail productif et reproductif entre conjoints. Cependant, cela n'empêche pas l'épouse de participer de manière irrégulière à l'activité de l'entreprise. Cette aide ponctuelle invisible, allant de soi, ne fait l'objet d'aucune reconnaissance ni valorisation. Enfin, une dernière configuration articule travail indépendant masculin et travail salarié féminin. Ici aussi, l'indépendance est une « affaire de couple » au sens où peut s'opérer une forme de complémentarité des statuts d'emploi des conjoints. I. BERTAUX-WIAME (2004, p. 31) parle à ce propos « d'un passage à l'indépendance sous condition de salariat ».

2. Il a fallu attendre les travaux plus récents portant sur les femmes ouvrières pour penser ensemble les rapports de genre au travail et au foyer (KergoAt 1982 ; Borzeix, MARUANI, 1982). 
Ces différentes configurations ont pour point commun que le travail des femmes y est en partie ou totalement invisible, subordonné et gratuit. Face à ce modèle du travail indépendant explicitement masculin et fortement conservateur quant aux rapports sociaux de sexe, on comprend que l'émancipation potentielle des femmes par le travail a surtout été pensée via le salariat. De ce fait, l'étude de la condition des femmes dans le monde des indépendants a été progressivement délaissée. Ainsi, Rose-Marie LAGRAVE (1987) regrette que les agricultrices, bien qu'ayant constitué dans les années 1970-1980 un sujet épistémologique décisif dans le champ des études féministes, n’aient plus fait l'objet de recherches au moment où, précisément, leur condition connaissait des transformations majeures, en particulier la consolidation législative de leur statut ${ }^{3}$.

Mais surtout, dans ces analyses des femmes dans le monde de l'indépendance, celles-ci ne sont jamais des cheffes d'entreprise. Si l'étude des entreprises familiales permet de saisir la domination professionnelle et domestique des femmes, elle laisse dans l'ombre les indépendantes qui, bien que minoritaires, forment depuis longtemps une part non négligeable des indépendant.e.s, surtout dans le commerce. Or deux transformations invitent à poursuivre et à renouveler ces investigations : l'indépendance connait un regain et des transformations importantes, nous l'avons dit ; la place des femmes y a radicalement changé, les aides familiales étant devenues extrêmement rares, comme nous allons le voir.

\section{D'aides familiales à cheffes d'entreprises : les transformations de la place des femmes dans l'indépendance}

Après plus d'un siècle de croissance quasi continue du salariat en France comme dans la plupart des pays occidentaux, le travail indépendant connait depuis quelques années un certain essor. Numériquement d'abord, puisque la part du travail indépendant dans l'emploi total remonte périodiquement depuis les années 1980 dans la majorité des pays de l'Organisation de coopération et développement économiques (OCDE) (ARUM, MÜLLER, 2004) et, en particulier quoique dans une moindre mesure, en France (Amossé, Goux, 2004 ; AbDELnour, 2017). Symboliquement ensuite, le travail indépendant fait l'objet d'un investissement renouvelé dans une société de travail déstabilisée par le chômage et par l'expansion de ce que l'Institut national de la statistique et des études économiques (Insee) désigne par « formes particulières d'emploi », participant d'un effritement du salariat (CASTEL, 1995). Ce renouveau se traduit par l'émergence de formes nouvelles de travail indépendant : multiplication des statuts de micro-entreprises, diffusion de l'auto-entrepreneuriat (ABDELNOUR, 2017) et de statuts hybrides (coopératives d'activité, franchises, etc.). Enfin, au modèle de

3. En 1982, est créé le « statut du conjoint artisan ou commerçant travaillant dans l'entreprise familiale », révisé en 2005 de sorte à ce que le conjoint soit obligé de choisir un statut (collaborateur, salarié ou associé). Sur l'évolution législative des statuts des conjoints d'indépendants, voir BESSIÈre, 2011 ; COMER, 2011 ; MAZAUD, 2013. 
l'indépendance de métier s'adjoint de plus en plus massivement une indépendance de services, ainsi qu'un développement des professions libérales (BEFFY, 2006). Alors que ces évolutions pourraient théoriquement faciliter l'accès des femmes à l'indépendance, qu'en est-il réellement?

Si l'on regarde les données disponibles depuis 1982, dans l'ensemble des nonsalarié.e.s, la part des femmes a légèrement diminué, passant de $36 \%$ en 1982 à $32 \%$ en 2012 (graphique 1). Mais ce constat, précédemment établi par Florent FAVRE (2009, p. 32), porte une vision familiale du non-salariat qui, précisément, mêle les indépendant·e.s (au sens d'employeur·euse-s et de travailleur.euse-s à leur compte) et leurs conjoint-e.s lorsqu'ils ou elles les aident dans leur travail (AmOssÉ, 2004), ce cas de figure étant comme on l'a vu majoritairement celui d'un homme à son compte et d'une conjointe aidante. De fait, les nomenclatures utilisées (celle des professions et des catégories socioprofessionnelles et celle des statuts d'activité) associent les aides familiaux-ales (et les conjoint·e-s collaborateur.rice-s) aux non-salariée.es - qu'il faut penser à exclure si l'on souhaite ne travailler que sur les individus juridiquement chefs d'entreprise et non pas sur l'ensemble des non-salarié.e.s. Pour saisir les transformations de la place des femmes dans l'indépendance et les rapporter aux dynamiques de la stratification sociale, on doit être attentif à l'histoire des catégorisations statistiques (ibid.). On évite alors de mêler celles qui travaillent, plus ou moins gratuitement, pour l'entreprise que dirige leur mari et celles qui dirigent elles-mêmes leur entreprise.

Or au sein du monde des non-salariée.e.s, la transformation la plus importante des trente dernières années est sans doute la très forte diminution des aides familiaux·ales, passant de près de 800000 en 1982 à environ 50000 en 2014 (graphique 2) ${ }^{4}$. Cette évolution contribue pour une grande partie à l' affaiblissement du poids des non-salarié.e-s dans la population active et redessine en profondeur la place qu'y occupent les femmes. Le visage du non-salariat, et donc de l'indépendance, a ainsi profondément changé : les aides familiaux ales représentaient $25 \%$ des emplois non salariés en 1982, contre seulement $3 \%$ aujourd'hui (graphique $3^{5}$ ) ; en 1982, $59 \%$ des femmes relevant du non-salariat étaient aides familiales, elles sont $8 \%$ à l'être aujourd' hui. Elles travaillent ainsi plus qu'avant pour leur propre compte et non, en tant qu' aide familiale, pour celui d'un père ou d'un conjoint. La légère baisse de la part des femmes dans le non-salariat masque en fait une nette progression des femmes parmi les indépendant.e.s.

Si l'on s'intéresse aux actif.ve-s qui sont eux.elles-mêmes à la tête de leur entreprise (ceux·elles que l'Insee catégorise comme indépendant·e·s, qu'ils·elles soient employeur.euse-s ou non), on constate en effet une tout autre évolution. D'après l'enquête Emploi, les femmes sont de plus en plus présentes parmi les chef·fe·s d'entreprise (graphique 1) : alors qu'elles représentaient $21 \%$ des indépendant·e.s sans main-d'œuvre en 1982 (et 17 \% des employeur-euse-s, c'est-à-dire qui ont au moins

4. Ces statistiques sont toutefois à manier avec précaution. Rose-Marie LAGRAVE (1987) a ainsi étudié le cas de « l'agricultrice inclassable », qui fait l'objet d'un flou statistique. Pour une critique approfondie de la mesure statistique du travail des femmes, voir MARUANi, Méron, 2012.

5. Les données reprises dans cette figure et dans ce paragraphe proviennent d'AMAR et al., 2016, p. 21. 
GRAPHIQUE 1 - La place des femmes parmi les actif·ve·s et les indépendant·e•s en 1982 et en 2012

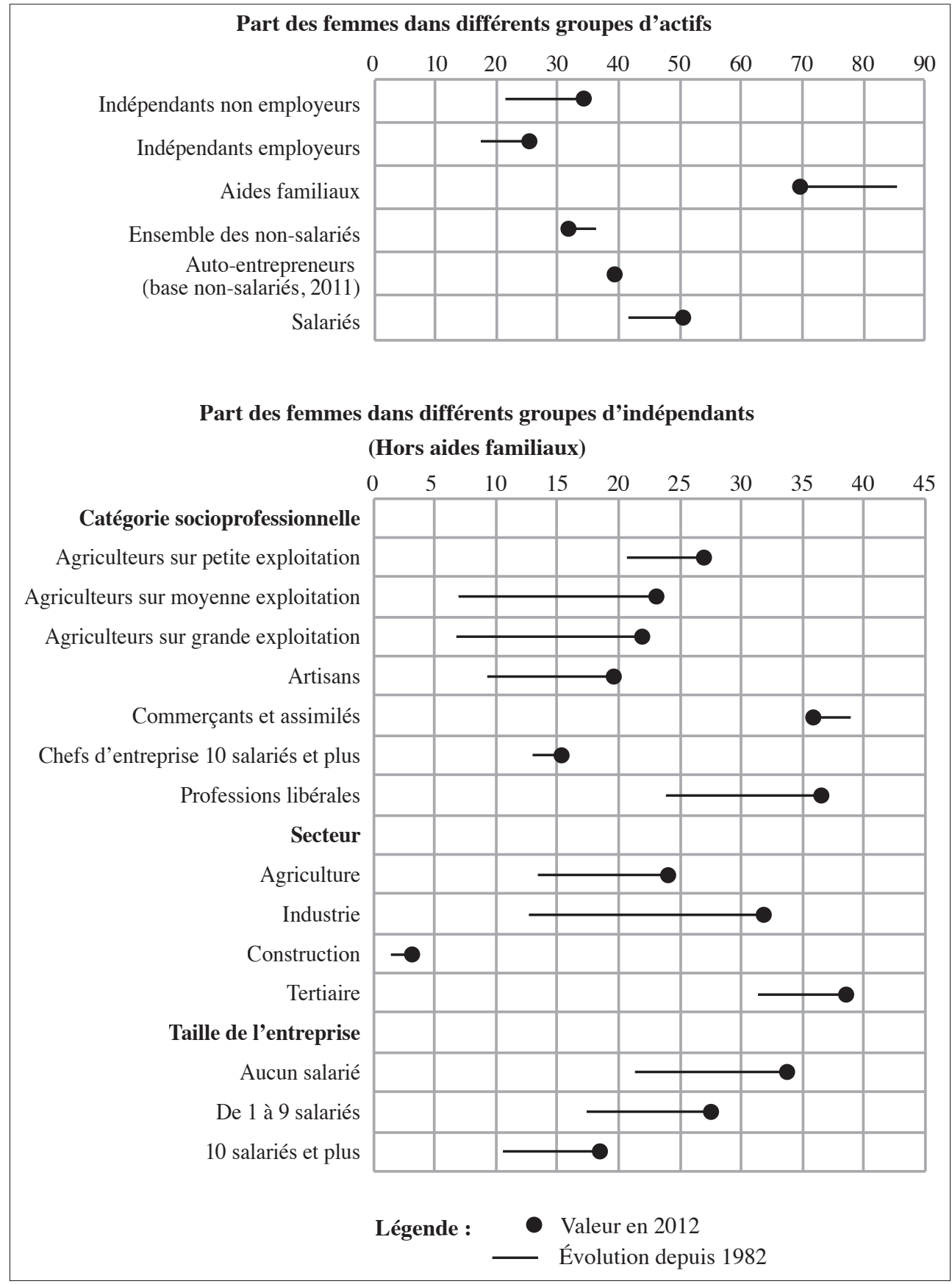

Note : par souci de lisibilité, les intervalles de confiance liés à l'échantillonnage des enquêtes Emploi n'ont pas été reportés. Lecture : en 2012, les femmes représentent 36,5\% des personnes travaillant en professions libérales contre $23,9 \%$ en 1982 . Champ : France métropolitaine.

Source : enquêtes Emploi 1982, 2012, Insee. 
GRAPHIQUE 2 - Effectifs des femmes et des hommes indépendant·e•s et aides familiaux•ales depuis 1982

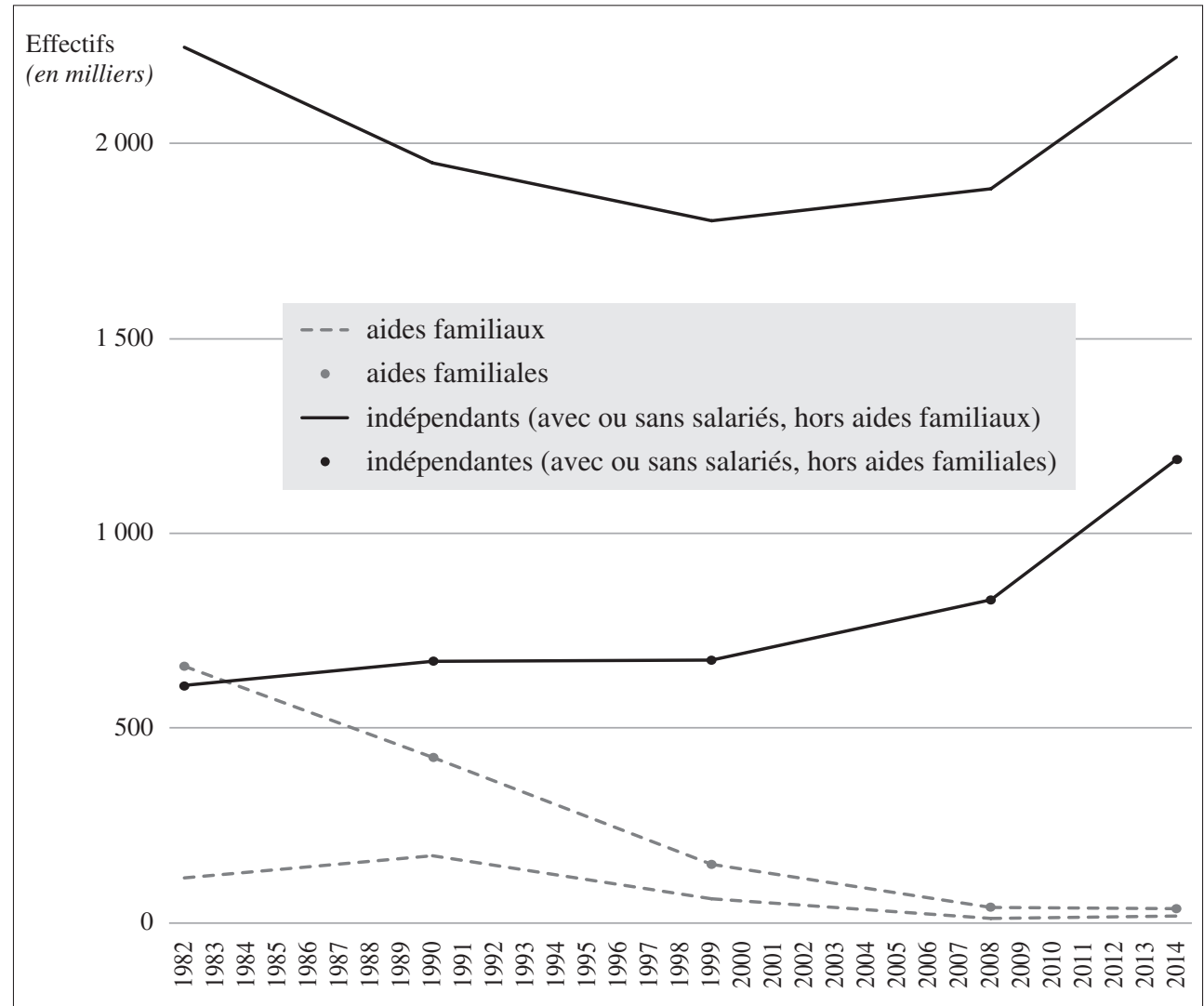

Champ : France métropolitaine, ensemble des non-salarié.e.s.

Source : recensements de la population (jusqu'à 2008, chiffres repris de MARUANI, MéRON, 2012), puis 2014, chiffres de l'Insee : https:// www.insee.fr/fr/statistiques/2011101?geo=FRANCE-1, consulté le 17 octobre 2017.

un·e salarié·e), elles en représentent $34 \%$ en 2012 (25\% pour les employeur·euse·s). Mais si les actif·ve·s à la tête d'une entreprise sont de plus en plus des femmes, cela se fait néanmoins dans des proportions variables selon les professions et les secteurs. Bien plus nombreuses qu'avant chez les agriculteur·rice·s (surtout parmi les plus important·e·s d'entre eux·elles) et parmi les artisan·e.s, elles le sont en revanche un peu moins parmi les commerçant·e·s, groupe déjà relativement féminin en 1982 (39 \%). Dans les fractions les plus hautes de la structure sociale, on constate deux tendances : la part des femmes parmi les chef.fe·s d'entreprise de plus de dix salarié.e.s n'a quasiment pas changé et reste faible, alors qu'il a explosé parmi les professions libérales, qui sont désormais le groupe d'indépendant·e·s le plus féminisé (près de $37 \%$ ).

Cette féminisation des chef·fe-s d'entreprise est particulièrement forte dans l'agriculture et dans l'industrie, secteurs où les femmes étaient rares au début des années 1980. Déjà relativement présentes dans le secteur tertiaire à cette époque, elles le sont 


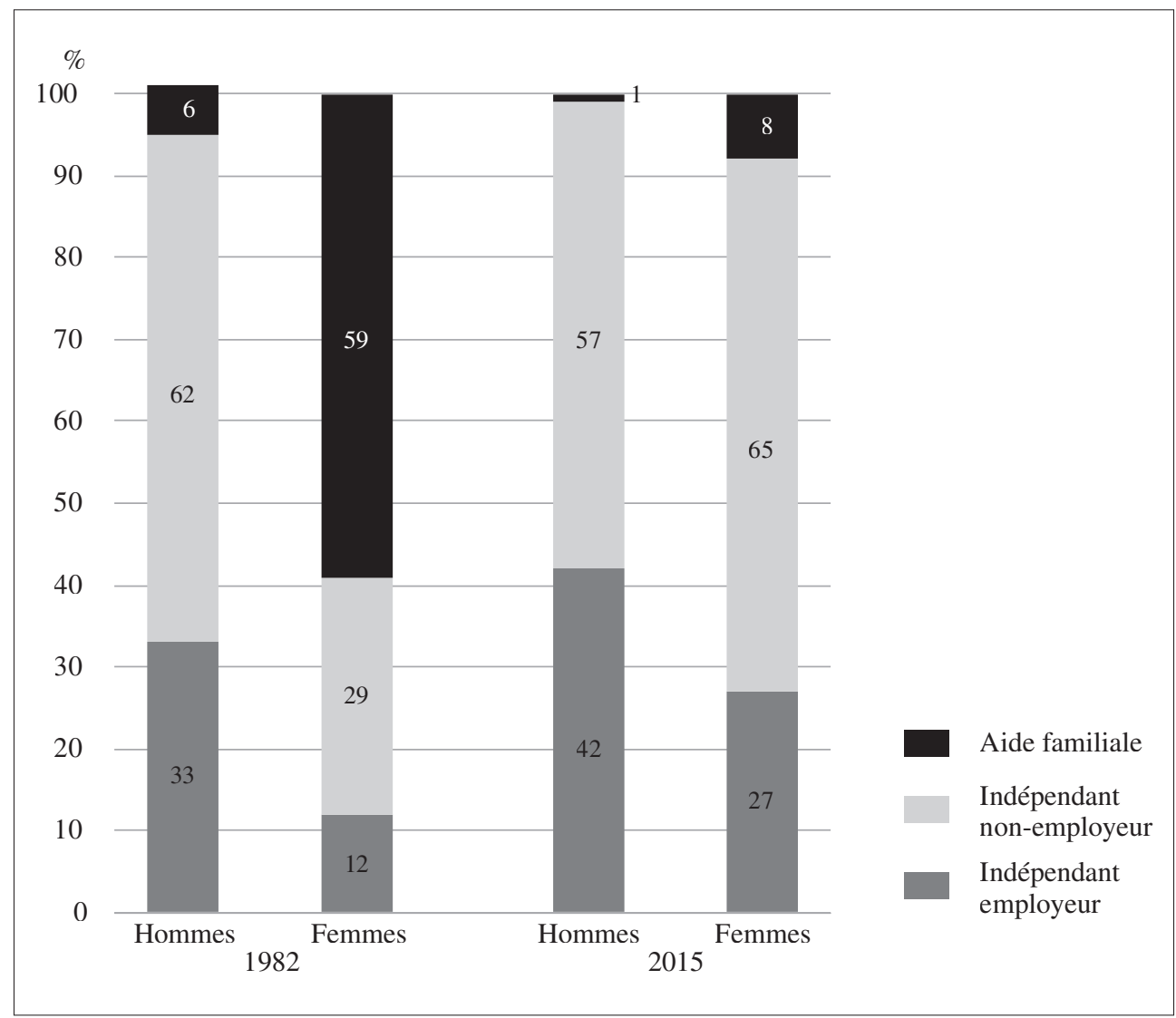

Champ : France métropolitaine, ensemble des non-salarié.e.s.

Source : enquêtes Emploi, Insee.

encore plus aujourd'hui - surtout dans le secteur de la santé et dans celui des services aux particuliers où elles représentent environ la moitié des chef·fe·s d'entreprises (OMALEK, RIOUX, 2015, p. 101). En revanche, si cette féminisation est assez importante et assez générale pour recomposer certains segments du monde des indépendant·e·s, elle change peu la hiérarchie des entreprises : à mesure que croît leur taille, les femmes sont moins nombreuses à les diriger. On comprend ainsi que l'auto-entrepreneuriat - entreprise individuelle dont la création est facilitée - attire des femmes dans une proportion plus forte (39\%) que la moyenne du non-salariat.

Dans certains cas, le travail en tant qu'indépendante des femmes reprend les traits de celui des hommes tel qu'il a été le plus documenté. Dans une enquête réalisée auprès d'une centaine de femmes cheffes d'entreprise de la région parisienne, Annie FouQueT (2005) observe d'ailleurs que l'élément déclencheur du projet de création d'entreprise est souvent l'association au projet d'un conjoint ou d'un autre 
membre de la famille. Nombre des enquêtées avaient des mères commerçantes ou des pères chefs d'entreprise. Elles partagent cette caractéristique avec l'ensemble des travailleur-se-s indépendant·e-s : $83 \%$ des agriculteur-rice-s ont au moins un parent indépendant et c'est le cas de $50 \%$ des chef.fe-s d'entreprise et de $45 \%$ des artisan.e.s et des commerçant.e.s (GollaC, 2009). En revanche, les conjointes d'indépendants viennent moins souvent d'une famille d'indépendants que les femmes elles-mêmes à la tête d'une entreprise (AMAR et al., 2016, p. 37). Cette reproduction sociale s'opère par le biais de formes variées de transmissions familiales, qui facilitent l'accès à l'indépendance professionnelle mais ce, davantage pour les hommes que pour les femmes (BESSIÈRE, 2010). En outre, ces dernières ont une possibilité moindre que les hommes de mobiliser la famille pour leur propre compte et semblent avoir besoin de davantage de ressources, notamment scolaires, pour se mettre à leur compte : leur niveau de diplôme est plus déterminant dans la probabilité de s'installer que pour leurs homologues masculins (RouAult, 2001). Dans ce cadre, il est envisageable que la tertiarisation de l'indépendance et l'essor récent de statuts hybrides et de nouvelles formes de non-salariat favorisent l'accès des femmes à l'indépendance, en leur permettant de s'installer à moindre coût économique et sans nécessairement de mobilisation familiale, mais au prix de chiffres d'affaires inférieurs. Les femmes participeraient ainsi au passage d'un modèle de l'indépendance par héritage dans lequel elles étaient largement perdantes à un modèle de création d'entreprise (FAVRE, 2009).

Cependant, il est tout aussi envisageable que l'accès plus facile des femmes à l'indépendance soit ambivalent et corresponde autant à des formes d'ascension sociale qu'à des déclassements. On sait, en effet, que la part du travail indépendant a tendance à augmenter en période de récession et de chômage (MARCHAND, 1998). Dans un contexte de précarisation et de chômage, les statuts hybrides tels que l'auto-entrepreneuriat peuvent être envisagés comme des compléments de revenu (LEVRATTO, SERVERIN, 2012) ou des « substituts au chômage » (ABDELNOUR, 2014). Les situations socio-économiques des non-salariés étant fortement différenciées, en ce qui concerne leurs revenus, leur patrimoine, leur niveau d'éducation, leurs conditions de travail et de santé (LAmarche, Romani, 2015 ; AlgaVA, VINCK, 2009 ; InSERM, 2011), l'activité croissante des femmes comme travailleuses indépendantes peut tout autant constituer un vecteur d'accès à des positions économiques dominantes qu'à de nouvelles modalités de la domination masculine au travail. On peut le supposer, les trajectoires de mises à son compte étant largement hétérogènes. D'un côté, les jeunes femmes investissent fortement les professions libérales juridiques et médicales (FAVRE, 2009), laissant voir un investissement par le haut de l'indépendance, ce qui n'empêche pas d'y retrouver les mêmes caractéristiques relatives à l'emploi des femmes : hiérarchisation et ségrégation sexuée, différenciation des modes d'exercice, des temps de travail et des revenus (LAPEYRE, 2006, à propos des avocates, des médecins et des architectes). De l'autre, elles sont plus présentes à la tête des plus petites entreprises dans le secteur des services dégageant de très faibles revenus. 
En dépit de la forte hétérogénéité du travail non salarié, ces positions méritent d'être pensées ensemble. En effet, au-delà des forts effets de composition qui structurent le monde des indépendants, on peut y observer des constantes qui nous semblent avoir un lien particulièrement étroit avec la différenciation sexuée du travail : horaires étendus (GolLAC, VOLKOFF, 2007) et forte perméabilité des espaces et des temps professionnels et domestiques. Si le salariat a pu être pensé comme le support de formes d'émancipation des femmes, c'est en raison de sa dimension régulée et de la distance qu'il introduisait avec la sphère domestique. L'interrogation sur les effets de l'indépendance féminine semble alors déterminante pour saisir les formes contemporaines de division sexuée du travail professionnel comme domestique.

\section{Hypothèses et enjeux du dossier}

Plusieurs difficultés se posent lorsque l'on cherche à rendre compte des inégalités entre les sexes à l'œuvre parmi les non-salarié.e-s. La plus évidente tient sans doute à la diversité des secteurs dans lesquels sont occupé.e-s ces travailleur-euse-s, secteurs qui connaissent des modes de régulation et de hiérarchies spécifiques. Dans ce dossier, les études de cas proposées permettent de raisonner à l'échelle d'un secteur afin de restituer ces spécificités.

Plus difficile à résoudre reste le problème de l'adéquation des outils forgés pour la mesure des inégalités au sein du salariat à l'analyse des indépendant·e.s. En effet, les études de genre ont élaboré des grilles de lecture des inégalités entre les sexes adaptées à l'univers salarial. C'est ainsi qu'ont pu être appréhendés les inégalités de salaire (Lemiere, Silvera, 2008), les freins dans les carrières (Guillaume, Pochic, 2007 ; LAUFer, 2004 ; MARRY, 2004), les discriminations, etc. Ont alors été mises en évidence les conditions organisationnelles de la reproduction des inégalités entre les sexes, les organisations régissant les carrières selon des normes masculines de comportement, au travail et en dehors. Les marqueurs de ces inégalités ne sont pas directement transposables au travail indépendant tant ils sont liés à la législation du travail : exposition différentielle au chômage, temps partiel et sous-emploi, inégalités salariales. C'est aussi le cas de concepts comme celui de « plafond de verre », limite supérieure invisible freinant les carrières des femmes, qui prennent appui sur les murs des organisations. Il faut donc d'autres outils pour penser les inégalités dans le travail indépendant des femmes et des hommes.

La tâche est d'autant plus difficile que l'on cherche à rendre compte des processus de domination à l'ouvre dans un espace dont on peine à décrire la stratification. Les revenus des indépendant·e.s ne sont pas comparables à ceux des salariéee·s et ne permettent pas toujours de mettre en évidence des hiérarchies internes ; le patrimoine (BEsSIÈrE et al., 2011) ou la qualification (AMOSSÉ, GouX, 2004) sont davantage appropriés pour différencier certaines catégories d'indépendant·e.s, mais le sont plus ou moins selon les secteurs, et rendent moins bien compte des nouvelles formes de 
travail indépendant, encore très peu régulées et concernant des populations moins dotées en patrimoine. L'une des manières de rendre compte des inégalités sexuées dans l'espace des indépendant-e.s est ainsi d'analyser les parcours professionnels dans lesquels intervient l'installation, de comprendre ce que la place particulière des femmes doit à des différences dans les modes d'accès et les manières d'être indépendant·e (activité adossée ou non à la carrière d'un·e conjoint·e) ainsi qu'à une segmentation sexuée des activités.

L'accès inégal des femmes et des hommes au statut d'indépendant peut provenir de normes et de socialisations qui associent moins les compétences nécessaires pour s'installer à son compte aux compétences féminines. Pourtant, dans le cas des classes populaires, on observe que les compétences associées au travail indépendant (comptabilité, gestion) sont davantage acquises et mobilisées par les femmes que par les hommes (PFEFFERKORN, 2004), et que les dispositions qu'il sollicite peuvent entrer en tension avec les socialisations masculines au travail manuel (GROS, 2015). Le «travail des signes » (VERRET, 1995, p. 41 et suivantes), celui des bureaux, est perçu négativement par les hommes ouvriers : ce sont des tâches contre lesquelles ils se définissent (WILLIS, 2011, p. 251 et suivantes). De façon cohérente, chez les artisans, l'aide familiale a généralement pris en charge une part du travail administratif de l'entreprise (JAEGER, 1982). Une analyse des différentes représentations de l'indépendance professionnelle selon le sexe et la classe sociale permet de comprendre les modalités qui conduisent aujourd'hui, de manière différenciée, femmes et hommes de milieux variés à s'installer à leur compte. En outre, l'accès inégal à l'indépendance professionnelle des femmes et des hommes reprend une division sexuée des activités et des professions. Les idéaux-types des indépendants - agriculteurs, artisans, commerçants, patrons - sont des figures masculines : l'émergence et l'institutionnalisation de groupes professionnels d'indépendants ont probablement une dimension sexuée encore mal explorée.

Derrière des manières différentes de devenir puis d'être indépendant·e, le déroulement des trajectoires professionnelles est différencié selon le sexe. Les indépendantes ont des revenus moins élevés que les indépendants, même si la mesure de ces inégalités est hasardeuse (FAVRE, 2009), précisément en raison du statut d'emploi, d'importants effets de composition (les femmes n'exercent pas les mêmes activités que les hommes) et d'une plus forte intrication des revenus des indépendant-e.s à ceux du ménage. Ainsi, en 2011, elles auraient retiré en moyenne 2070 euros nets par mois de leur activité non salariée, soit $28 \%$ de moins que les hommes (OMALEK, Rioux, 2015, p. 100) ${ }^{6}$. Plus que la hiérarchie des secteurs et des activités, c'est la valeur économique et symbolique

6. Ces écarts bruts doivent être maniés avec précaution et décomposés pour une meilleure compréhension des processus, ce que rappelle Florent FAVRE (2009, p. 31) sur des données légèrement plus anciennes : « Le revenu moyen des hommes non salariés est de $40 \%$ supérieur à celui des femmes. Cet écart varie avec certains facteurs tels que le secteur d'activité, l'ancienneté, l'âge ou encore la taille de l'entreprise dirigée. En outre, la prise en compte d'une durée du travail des non-salariées plus faible que celle de leurs confrères masculins rapprocherait cet écart de celui constaté pour les salariés travaillant à temps complet, soit $23 \%$. » 
du travail réalisé qui semble faire l'objet de redéfinitions sexuées. En particulier, puisque ni la durée ni la rémunération du travail ne sont déterminées juridiquement, les mécanismes qui permettent d'accroître le chiffre d'affaires d'une entreprise sont très différents de ceux qui régissent les carrières salariales. Les hiérarchies et les rapports de domination qui jouent dans la répartition des revenus (ampleur du travail fourni, valeur monétaire de ce travail, position dans la concurrence) sont, en théorie, sans cesse redéfinis par le jeu du marché, et ne sont pas aussi visibles que dans le cas d'activités salariées. Ils jouent pourtant directement dans les trajectoires, et impliquent des savoir-faire relationnels et des ressources dont on peut supposer qu'ils sont inégalement distribués entre femmes et hommes (relations aux fournisseurs, aux clients).

\section{Parcours de lecture}

L'origine de ce dossier réside dans une journée d'étude organisée en 2015 au cours de laquelle ont été présentées, outre celles que l'on peut lire ici, des recherches sur les femmes dans le monde masculin de l'artisanat (Olivier Crasset), l'entrepreneuriat féminin en Guyane française (Dorothée Serges-Garcia), l'accès des chômeuses à l'indépendance via le statut d'auto-entrepreneur (Guillaume Blache, Murielle Matus et Nicolas Prokovas), le rôle donné au genre dans la justification des faillites d'entreprises (Virginie Blum), l'engagement politique des agricultrices (Clémentine Comer) et les femmes créatrices d'entreprises de conseil en égalité professionnelle (Soline Blanchard). Le fait que la quasi-totalité de ces interventions aient porté sur des femmes cheffes d'entreprises et peu (bien moins que ce à quoi nous nous attendions) sur la place des femmes dans des entreprises familiales est à nos yeux révélateur des transformations profondes qu'a connues le travail indépendant ces dernières décennies.

Ce dossier est l'occasion de revisiter l'articulation entre genre et travail indépendant, et ce d'abord par l'analyse de différentes figures féminines du non-salariat contemporain, à travers trois études de cas : les artisanes d'art, les agricultrices néorurales et les mompreneurs. Au travers de ces cas, les articles mettent en évidence les transformations de la place occupée par les femmes dans le travail indépendant, ainsi que les usages ambivalents qu'elles font de ce statut. Pour clore le dossier, une analyse statistique du temps de travail des indépendantes montre d'ailleurs les inégalités, en particulier en termes de gestion du travail domestique, que dessine aujourd'hui le non-salariat.

La contribution d'Anne Jourdain permet d'approcher ces questions à travers la féminisation de l'artisanat d'art. Derrière la croissance du taux de féminisation de ces professions se profilent des différenciations sexuées dans l'accès et dans l'exercice du métier : d'une part, les femmes sont plus souvent d'anciennes cadres reconverties ; d'autre part, elles valorisent essentiellement la dimension créative de leur activité. Femmes et hommes ne pratiquent pas leur travail de la même manière, n'en valorisent pas les mêmes aspects et n'y consacrent pas le même temps. Anne Jourdain montre que 
cette différenciation va de pair avec une hiérarchisation des produits et des marchés. Le résultat de son enquête est alors surprenant. Les femmes sont davantage situées sur le pôle artiste des artisan.e.s d'art, ce qui aurait pu donner lieu à une valorisation accrue de leur production. Mais, paradoxalement, ce positionnement tend à délégitimer les artisanes d'art dans le métier. L'auteure apporte ainsi une contribution importante à l'analyse des liens entre féminisation et (dé)valorisation de l'activité. Si l'artisanat se trouve reconfiguré par sa féminisation, les inégalités se déplacent plus qu'elles ne s'effacent.

Le monde du travail non salarié constitue un laboratoire pour mettre en évidence le rôle du genre dans la définition de la valeur de ce que produit un individu, selon son statut d'emploi, son sexe et les ressources dont il dispose. Plus largement, nous avons souhaité prendre au sérieux l'effet du genre à la fois dans la formation et la recomposition des frontières juridiques qui structurent la division du travail ${ }^{7}$ et dans la répartition des femmes et des hommes dans ces espaces aux valeurs différentes. Pour cela, il faut admettre qu'une partie des inégalités entre femmes et hommes est liée à l'ordre domestique inégalitaire en tant qu'il détermine et est déterminé par, de fait ou supposément, les modalités de l'engagement au travail et de l'émancipation des femmes.

Pour explorer le renouvellement des exploitations agricoles conjugales à une époque où les aides familiales sont devenues rares, la contribution de Madlyne Samak interroge les modes d'installation des «néo-ruraux » à travers le cas des maraîchers biologiques des Alpes-Maritimes. Elle démontre que si les inégalités de genre se sont globalement réduites dans l'agriculture, elles se sont en revanche accrues dans le groupe des «néo-ruraux ». Alors que l'on pourrait intuitivement penser que ces exploitations agricoles, en mobilisant des personnes issues des classes moyennes ou supérieures, urbaines et diplômées, seraient un lieu de réinvention des normes de genre, elles révèlent paradoxalement la permanence des inégalités, voire leur résurgence. Si les installations se font dans un cadre de reconversions professionnelles, c'est le plus souvent en couple et à l'intiative des hommes. Le projet individuel de reconversion devient alors un projet de couple qui se déploie en défaveur des femmes, qui exercent leur activité largement sans statut. L'acceptation de ces inégalités (conditions de travail difficiles, rémunérations faibles), au sein d'une population jeune et relativement diplômée ailleurs, plus portée à les contester, se fait au nom d'une solidarité conjugale face aux difficultés de survie d'exploitations aux chiffres d'affaires modestes. De façon surprenante, une forme de politisation permet de justifier ces inégalités : la défiance de ces « néo-ruraux » envers les institutions favorise l'acceptation du vide statutaire des femmes, du moins tant que le couple dure.

Dans son article, Julie Landour se concentre quant à elle sur des formes de travail indépendant exclusivement féminin en s'intéressant à la figure des mompreneurs.

7. Travail salarié délimité par un contrat de travail, travail domestique légalement non rémunéré, travail indépendant légalement rémunéré hors d'un contrat de travail, travail au noir illégalement rémunéré. 
L'auteure a interrogé des membres de collectifs revendiquant ce label qui désigne des femmes, et plus particulièrement des mères, se mettant à leur compte pour mieux concilier leur vie professionnelle et leur vie familiale. L'analyse conjointe des trajectoires et des discours de ces femmes permet de penser les deux dimensions de leur installation comme indépendantes : les difficultés éprouvées durant leur expérience salariale et la valorisation de l'entrepreneuriat. Ainsi ces femmes se sont-elles senties empêchées ou bloquées dans leur carrière salariée, notamment en raison de leur engagement parental. L'indépendance est alors envisagée comme la solution qui permet de concilier l'investissement dans les deux sphères. Au-delà de ces aspirations, l'article montre bien les tensions qui traversent les situations de ces mompreneurs. Bien qu'elles souhaitent s'y engager, on voit comment leur activité professionnelle se trouve en pratique marginalisée par rapport à un travail domestique et parental intense. Cet engagement professionnel n'est d'ailleurs pas dénué de risque pour ces femmes, les séparations conjugales fonctionnant alors comme un puissant révélateur de la fragilité de leur position sociale et matérielle.

Appuyée sur des données statistiques nationales, la contribution d'Amandine Barrois et de François-Xavier Devetter démontre que la « conciliation » de la carrière professionnelle avec l'engagement parental permise par le statut non salarié est un leurre. Alors même que s'impose un discours vantant les mérites de l'entrepreneuriat pour les femmes de ce point de vue, la comparaison du temps de travail des femmes salariées et non salariées montre une tout autre réalité. Les auteurs mettent ainsi en évidence les spécificités du temps de travail des indépendantes, qui se révèlent contradictoires avec une logique de « conciliation ». Leurs durées du travail sont en effet longues, leurs horaires irréguliers et imprévisibles. Ils observent également une hétérogénéité en fonction du niveau de qualification. Ainsi, quand la « liberté du temps de travail » se traduit pour les plus qualifiées par des difficultés accrues à articuler temps de travail et temps « hors-travail », elle se solde pour les moins qualifiées par de faibles rémunérations assimilées à une nouvelle forme de salaire d'appoint.

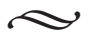

Ces recherches s'accordent sur un constat : les évolutions de l'indépendance professionnelle s'accompagnent d'une reformulation des inégalités de genre. Persistantes, voire réaffirmées, ces inégalités revêtent plusieurs formes : l'invisibilisation d'une partie du travail fourni, la faible formalisation de certains statuts, l'emprise du travail domestique sur l'activité professionnelle, dont elle est mal dissociée. Les unes s'investissent dans un projet conjugal mené par l'homme, les autres assument, très souvent seules, leur projet d'installation. Les situations de travail indépendant étudiées dans ce numéro démontrent en effet que cette forme d'emploi autonome n'est pas davantage source d'émancipation pour les femmes que le salariat. L'asymétrie de la mobilisation conjugale autour d'une activité invasive comme l'est l'indépendance professionnelle demeure, et elle traverse la structure sociale. On la retrouve ainsi dans les professions 
dominantes où l'impératif d'être « une bonne mère » conduit les femmes à réinvestir dans leur vie familiale des compétences professionnelles managériales, déléguant au marché les tâches les moins valorisantes pour allouer leur temps domestique au travail d'éducation des enfants (RABIER, 2013). Néanmoins, un angle mort de ce dossier permettrait peut-être de nuancer ce constat. Les professions libérales, offrant plus de stabilité et mieux dissociées de l'espace et des économies domestiques, pourraient souligner, en creux, quelques formes d'atténuation des inégalités de genre que rendraient possible la régulation des statuts et l'accumulation des ressources lorsque l'on atteint le haut de la hiérarchie des professions.

\section{BIBLIOGRAPHIE}

AbDELNOUR S. (2014), «L'auto-entrepreneuriat : une gestion individuelle du sous-emploi », Nouvelle Revue du travail [en ligne], $\mathrm{n}^{\circ} 5$ : http://nrt.revues.org/1879, consulté le 13 octobre 2017.

AbDelnour S. (2017), Moi, petite entreprise. Les auto-entrepreneurs, de l'utopie à la réalité, Paris, Presses universitaires de France.

Algava É., VincK L. (2009), « Les conditions de travail des non-salariés », Premières Synthèses, $\mathrm{n}^{\mathrm{o}} 50.1$, Direction de l'animation de la recherche, des études et des statistiques (Dares).

AMAR N., LAFFOn P., LE Minez S. (2016), La Protection sociale des non-salariés et son financement. Rapport, Paris, Haut Conseil du financement de la protection sociale.

Amossé T. (2004), « Professions au féminin. Représentation statistique, construction sociale », Travail, genre et sociétés, $\mathrm{n}^{\circ} 11$, pp. 31-46.

Amossé T., Goux D. (2004), "Entries and Exits from Self-Employment in France over the Last Twenty Years", in Arum R., Müller W., The Reemergence of Self-Employment, Princeton/ Oxford, Princeton University Press, pp. 75-103.

ARUM R., MüLlER W. (2004), The Reemergence of Self-Employment: A Comparative Study of Self-Employment Dynamics and Social Inequality, Princeton/Oxford, Princeton University Press.

BARThez A. (1982), Famille, travail, agriculture, Paris, Economica.

Battagliola F. (2000), Histoire du travail des femmes, Paris, La Découverte.

BEFFy M. (2006), « Moins d'artisans, des professions libérales en plein essor », in Insee, France, portrait social. Édition 2006, Paris, Insee, coll. «Insee Références », pp. 139-156.

Bereni L., Chauvin S., Jaunait A., Revillard A. (2008), Introduction aux gender studies. Manuel des études sur le genre, Paris, De Boeck.

BertauX-Wiame I. (1982), «L'installation dans la boulangerie artisanale », Sociologie du travail, vol. 24, $\mathrm{n}^{\circ} 1$, pp. 8-23.

BertauX-Wiame I. (2004), « Devenir indépendant, une affaire de couple », Cahiers du genre, $\mathrm{n}^{\mathrm{o}} 37$, pp. 13-40. 
BessiÈRE C. (2010), De génération en génération. Arrangements de famille dans les entreprises viticoles de Cognac, Paris, Raisons d'agir.

BESSIÈRE C. (2011), «Les séparations conjugales dans les familles agricoles », Informations sociales, $\mathrm{n}^{\circ} 164$, pp. 64-71.

Bessière C., Paoli (de) C., Gouraud B., Roger M. (2011), «Les agriculteurs et leur patrimoine : des indépendants comme les autres ? », Économie et statistique, $\mathrm{n}^{\circ}$ 444-445, pp. 55-74.

BorzeIX A., MARUANi M. (1982), Le Temps des chemises. La grève qu'elles gardent au cour, Paris, Syros.

CASTEl R. (1995), Les Métamorphoses de la question sociale. Une chronique du salariat, Paris, Fayard.

Comer C. (2011), « La “conjointe collaboratrice” : un recul statutaire ambigu », Pour, $\mathrm{n}^{\circ} 212$, pp. 19-24.

DelPhy C. (1983), «Agriculture et travail domestique : la réponse de la bergère à Engels », Nouvelles Questions féministes, $\mathrm{n}^{\circ}$ 5, pp. 3-17.

DelPhy C. ([1978], 1998), « Travail ménager ou travail domestique ? », in Delphy C., L'Ennemi principal. 1, Économie politique du patriarcat, Paris, Syllepse, pp. 57-73.

FAVRE F. (2009), « Hommes-femmes, des différences de revenu sensibles pour les non-salariés », in Insee, Les Revenus d'activité des indépendants. Édition 2009, Paris, Insee, coll. «Insee références », pp. 31-45.

Fouquet A. (2005), «Les femmes chefs d'entreprise : le cas français », Travail, genre et sociétés, $\mathrm{n}^{\mathrm{o}} 13$, pp. 31-50.

Gollac M., Volkoff S. (2007), Les Conditions de travail, Paris, La Découverte.

Gollac S. (2003), « Maisonnée et cause commune : une prise en charge familiale », in Weber F., Gojard S., Gramain A. (dir.), Charges de famille. Dépendance et parenté dans la France contemporaine, Paris, La Découverte, pp. 274-311.

GollaC S. (2009), « Travail indépendant et transmissions patrimoniales : le poids des inégalités au sein des fratries », Économie et statistique, $\mathrm{n}^{\mathrm{0}}$ 417-418, pp. 55-75.

GRESLE F. (1981), L'Univers de la boutique. Famille et métier chez les petits patrons du Nord (1920-1975), Lille, Presses universitaires de Lille.

Gros J. (2015), Des classes populaires à la lisière du salariat. Une analyse des bûcherons entre emploi, marché et stratification sociale, thèse de doctorat en sociologie, Paris, École des hautes études en sciences sociales.

Guillaume C., Pochic S. (2007), « La fabrication organisationnelle des dirigeants. Un regard sur le plafond de verre », Travail, genre et sociétés, $\mathrm{n}^{\circ}$ 17, pp. 79-103.

INSERM (Institut national de la santé et de la recherche médicale) (2011), Stress au travail et santé : situation chez les indépendants. Synthèse et recommandations. Expertise collective, Paris, Inserm.

JAEGER C. (1982), Artisanat et capitalisme, l'envers de la roue de l'histoire, Paris, Payot. 
Kergoat D. (1982), Les Ouvrières, Paris, Le Sycomore

LAGRAVE R.-M. (1983), « Bilan critique des recherches sur les agricultrices en France », Études rurales, $\mathrm{n}^{\circ} 92$, pp. 9-40.

LAGRAVE R.-M. (1987), « L'agricultrice inclassable : les fonctions sociales du flou statistique », in Lagrave R.-M. (dir.) (1988), Celles de la terre. Agricultrice : l'invention politique d'un métier, Paris, Éditions de l'École des hautes études en sciences sociales, pp. 87-110.

LAMARCHE P., RomAni M. (2015), « Le patrimoine des indépendants », in Omalek L., Rioux L. (coord.), Emploi et revenus des indépendants, Paris, Insee, coll. « Insee références », pp. 75-90.

Lapeyre N. (2006), Les Professions face aux enjeux de la féminisation, Toulouse, Octarès.

LAUFER J. (2004), « Femmes et carrières : la question du plafond de verre », Revue française de gestion, $\mathrm{n}^{\mathrm{o}} 151$, p. 117-127.

LEMière E., SiLVera R. (2008), «Les différentes facettes des inégalités de salaires entre hommes et femmes », in Cornet A., Laufer J., Belghiti-Mahut (coord.), GRH et genre. Les défis de l'égalité hommes-femmes, Paris, Vuibert, pp. 139-158.

LEVRATto N., SERVERIN É. (2012), «L'auto-entrepreneur, instrument de compétitivité ou adoucissant de la rigueur? Bilan de trois années de fonctionnement du régime », Revue de la régulation [en ligne], n ${ }^{\circ} 12$ : https://regulation.revues.org/9879, consulté le 17 octobre 2017.

MARCHAND O. (1998), « Salariat et non-salariat dans une perspective historique », Économie et statistique, $\mathrm{n}^{\mathrm{O}}$ 319-320, pp. 3-11.

MARRY C. (2004), Les Femmes ingénieurs, une révolution respectueuse, Paris, Belin.

Maruani M., Méron M. (2012), Un siècle de travail des femmes en France (1901-2011), Paris, La Découverte.

MAZAUD C. (2013), L'Artisanat français. Entre métier et entreprise, Rennes, Presses universitaires de Rennes.

Omalek L., Rioux L. (coord.) (2015), Emploi et revenus des indépendants, Paris, Insee, coll. « Insee références ».

PfEFFERKoRn R. (2004), « Filières de formation sexuées, métiers "féminins" et politiques de mixité professionnelle », in Rogers R. (dir.), La Mixité dans l'éducation : enjeux passés et présents, Lyon, ENS éditions, pp. 573-607.

RABIER M. (2013), Entrepreneuses de cause. Contribution à une sociologie des engagements des dirigeantes économiques en France, thèse de doctorat en sociologie, École des hautes études en sciences sociales.

Rouault D. (2001) «Les revenus des indépendants et dirigeants : la valorisation du bagage personnel », Économie et statistique, $\mathrm{n}^{\circ} 348$, pp. 35-59.

SCHWEITZER S. (2002), Les femmes ont toujours travaillé. Une histoire du travail des femmes aux XIX ${ }^{e}$ et $X X^{e}$ siècles, Paris, Odile Jacob.

Verret M. (1995), Chevilles ouvrières, Paris, Les Éditions de l'Atelier/Les Éditions Ouvrières. 
TÉNÉdos J., Weber F. (2006), L'Économie domestique. Entretien avec Florence Weber. Ethnographie du quotidien 1, La Courneuve, Aux Lieux d'Être.

WeBer F. (1989), Le Travail à-côté. Étude d'ethnographie ouvrière, Paris, Institut national de la recherche agronomique, École des hautes études en sciences sociales.

WILLIS P. (2011), L'École des ouvriers. Comment les enfants d'ouvriers obtiennent des boulots d'ouvriers, Marseille, Agone.

ZALC C. (2005), « Femmes, entreprises et dépendances. Les entrepreneuses étrangères à Paris dans l'entre-deux-guerres », Travail, genre et sociétés, $\mathrm{n}^{\mathrm{o}}$ 13, pp. 51-74.

ZARCA B. (1986), L’Artisanat français. Du métier traditionnel au groupe social, Paris, Economica. 